\title{
miRNA Expression Profile of Saliva in Subjects of Yang Deficiency Constitution and Yin Deficiency Constitution
}

\author{
Yu Chen ${ }^{\mathrm{a}, \mathrm{c}}$ Yanling Wu ${ }^{\mathrm{b}}$ Haiqiang Yao ${ }^{\mathrm{a}}$ Hui Luo ${ }^{\mathrm{a}}$ Bing Lin ${ }^{\mathrm{c}}$ Xiuping Zhang ${ }^{\mathrm{a}}$ \\ Xue Lianga ${ }^{\mathrm{a}}$ Ranran Sun ${ }^{\mathrm{a}}$ Shipeng Zhao ${ }^{\mathrm{a}}$ Yingshuai Lia Yanfei Zhenga \\ Lingru Lia Qi Wanga
}

aSchool of traditional Chinese Medicine, Beijing University of Chinese Medicine, Beijing, ${ }^{\mathrm{b} D e p a r t m e n t}$ of Integrated Traditional and Western Medicine, People's Hospital of Wuhai Inner Mongolia, Wuhai, 'Department of Nutrition, China-Japan Friendship Hospital, Beijing, China

\section{Key Words}

Tcm constitution $\bullet$ Saliva $\cdot$ miRNA $•$ Target genes $•$ KEGG

\begin{abstract}
Background/Aims: Based on the theory of constitution in Traditional Chinese Medicine (TCM), the Chinese Han population has been classified into nine constitutions. Of these, Yang deficiency constitution mainly exhibit cold intolerance while Yin deficiency constitution mainly exhibit heat intolerance. Some studies have been carried out to explore the modern genetic and biological basis of such constitution classification, but more remains to be done. MicroRNA (miRNA) serves as post-transcriptional regulators of gene expression and may play a role in the classification process. Here, we examined miRNA expression profile of saliva to further improve the comprehensiveness of constitution classification. Methods: Saliva was collected from Chinese Han individuals with Yang deficiency, Yin deficiency and Balanced constitutions ( $n=5$ each), and miRNA expression profile was determined using the Human miRNA OneArray ${ }^{\circledR} v 7$. Based on 1.5 Fold change, means $\log _{2} \mid$ Ratio $\mid \geq 0.585$ and $P$-value $<0.05$, differentially expressed miRNA was screened. Target genes were predicted using DIANATarBasev7.0 and analysis of KEGG pathway was carried out using DIANA-mirPathv.3. Results: We found that 81 and 98 differentially expressed miRNAs were screened in Yang deficiency and Yin deficiency constitution, respectively. Among them, 16 miRNAs were identical and the others were unique. In addition, the target genes that are regulated by the unique miRNAs were significantly enriched in 27 and 20 signaling pathways in Yang deficiency and Yin deficiency constitution, respectively. Thyroid hormone signaling pathway is present in both constitutions. These unique miRNAs that regulated target genes of thyroid hormone signaling pathway may be associated with cold intolerance or heat intolerance. Conclusion: The results of our study show that Yang deficiency and Yin deficiency constitutions exhibit systematic differences in miRNA expression profile. Moreover, the distinct characteristics of TCM constitution may be explained, in part, by differentially expressed miRNAs.

Y. Chen and Y. Wu contributed equally to this work.

\begin{tabular}{ll}
\hline Lingru Li & School of traditional Chinese Medicine, Beijing University of Chinese Medicine \\
and Qi Wang & Beijing 100029 (China) \\
& Tel. +861064286766, E-Mail lilingru912@163.com; wangqi710@126.com
\end{tabular}




\section{Cellular Physiology Cell Physiol Biochem 2018;49:2088-2098

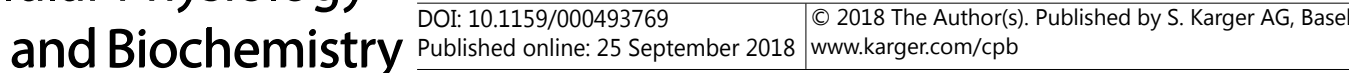 \\ Chen et al.: mRNA Expression Profile of Saliva in Yang/Yin Deficiency Constitution}

\section{Introduction}

The orientation of modern medicine has been shifting from disease to health in recent years. The medical model characterized by prediction, prevention, personalization and participation has gained greater prominence [1]. Some research has explored the individual differences between healthy population and associated disease risks [2, 3]. However, it is not clear whether the normal healthy population, within a specific ethnic identity, can be classified into distinctive groups, where each group exhibits its own biological features and disease risks. Based on TCM theory, healthy individuals have been classified into distinctive groups. The term "constitution" was coined to refer to this unique entity. The TCM constitution is an objective and existential life phenomenon. It is a comprehensive and relatively stable trait that manifests in morphological and physiological function and mental state that is based on congenital inheritance and latter-day acquisition throughout an individual's life [4]. "Constitution being separable", "relationship between constitution and disease" and "constitution being recuperable" are the three key scientific problems raised in TCM constitution [5]. Of them, accurate and objective classification is crucial. There are nine constitutions in the Chinese Han population [4]. The Yang deficiency constitution is mainly characterized by cold intolerance while the Yin deficiency constitution is mainly characterized by heat intolerance [4]. A series of studies have been conducted to investigate the genetic and biological basis related to their differences. Yang deficiency constitution may be correlated to hypothalamic-pituitary-adrenal and hypothalamic-pituitary-thyroid axes, and also to disorders of the cyclic nucleotide and immune systems [6]. A study of metabonomics showed that Yang deficiency constitution exhibited disorders in energy, lipid, glucose and amino acid metabolism, as well as impaired organ function [7]. Single nucleotide polymorphisms (SNPs) in the PPARD, PPARG, and APM1 genes were shown to be associated with constitutions of both Yang deficiency and Yin deficiency [8]. A study using peripheral blood mononuclear cells (PBMCs) showed that expression profiling-based clustering of constitutional subjects largely overlaps with TCM classification and is consistent with the clinical observation that subjects with Yang deficiency tended towards obesity. Seriesclustering analysis has also detected several key lipid metabolic genes to be down- and upregulated in Yin deficiency and Yang deficiency constitution, respectively [9]. These studies have provided clues for the objective classification into either of these constitutions.

Previous studies on TCM constitution mainly used blood samples since it is thought that blood can represent the overall characteristics of the human body. However, the use of blood samples is sometimes problematic due to inconvenience, its invasive nature, and the risk of infection. Moreover, the concept of TCM constitution is a comprehensive one and the studies using blood samples may yield an incomplete picture. As a result, other samples such as saliva and urine could be used for more a convenient and compete exploration. Due to the number of molecular substances it contains, saliva could be a good reflection of the physiological and pathological conditions in the human body. Compared with other samples, collection of saliva is convenient, non-invasive, economical and not influenced by the menstrual cycle. For this reason, some studies have focused on using saliva to detect diseases and have showed some clinical benefits $[10,11]$. Similarly, saliva could be used to improve the comprehensiveness of studies on TCM constitution.

miRNAs are endogenous, small, non-coding, single-stranded RNAs that serve as posttranscriptional regulators of gene expression [12]. By targeting the mRNAs of protein-coding genes, miRNAs play a critical role in a variety of biological and pathological processes [1316]. As a result, using miRNAs as biomarkers have received widespread attention [17-19]. While most miRNAs are found intracellularly, a significant number have also been observed extracellularly in saliva, urine and breast milk [20-23]. These miRNAs are stable and show distinct expression profiles [24]. Earlier work by our team has constructed miRNA expression profile of PBMCs in phlegm dampness constitution and screened the differentially expressed miRNAs. Systematic difference in the miRNA expression has been used in the objective classification of constitutions. The amount and color of saliva in Yang deficiency and Yin deficiency constitutions are different according to clinical presentations [4]. Differential expressions of miRNAs may exist in the saliva and play a potential role in the classification of TCM constitution. 


\section{Cellular Physiology Cell Physiol Biochem 2018;49:2088-2098

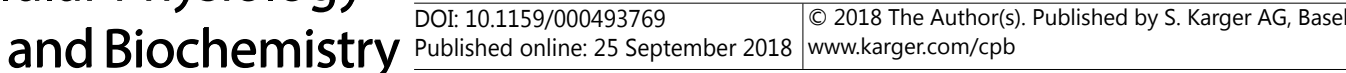 \\ Chen et al.: mRNA Expression Profile of Saliva in Yang/Yin Deficiency Constitution}

\section{Materials and Methods}

\section{Subjects and Saliva Sample Collection}

Subjects from the physical examination center of China-Japan Friendship Hospital and Hong Yi Tang of TCM were enrolled between September 2014 and December 2015. Individuals of balanced constitution (group P), Yang deficiency constitution (group Ya) and Yin deficiency constitution (group Yi) were chosen using a standardized questionnaire (Wang Qi's Body Constitution Classification Questionnaire-Chinese version, ZYYXH/T 157-2009) (Table 1, Table 2). A total of 15 subjects were finally included, with 5 in each

Table 1. Wang Qi's Body Constitution Classification Questionnaire. Note: [No] Never happened in the past year. [Slightly] It happened occasionally in the past year. [Sometimes] It happened Sometimes, but no regular pattern. [Often] It happened most of the time in the past year. [All the time] It happened all the time in the past year

\begin{tabular}{|c|c|c|c|c|c|}
\hline Experience/condition in the past year & No & Slightly & Sometimes & Often & $\begin{array}{l}\text { All the } \\
\text { time }\end{array}$ \\
\hline (1)Were you energetic? & 1 & 2 & 3 & 4 & 5 \\
\hline (2)Did you get tired easily? & 1 & 2 & 3 & 4 & 5 \\
\hline (3)Did you experience shortness of breath? & 1 & 2 & 3 & 4 & 5 \\
\hline (4)Did you get palpitations? & 1 & 2 & 3 & 4 & 5 \\
\hline (5)Did you get dizzy easily or become dizzy when standing up? & 1 & 2 & 3 & 4 & 5 \\
\hline (6)Did you prefer quietness and not like to talk? & 1 & 2 & 3 & 4 & 5 \\
\hline (7)Was your voice weak when talking? & 1 & 2 & 3 & 4 & 5 \\
\hline (8)Did you feel in low spirits and depressed? & 1 & 2 & 3 & 4 & 5 \\
\hline (9)Did you easily feel anxious and worried? & 1 & 2 & 3 & 4 & 5 \\
\hline (10)Did you feel overly sensitive, vulnerable or emotionally upset? & 1 & 2 & 3 & 4 & 5 \\
\hline (11)Were you easily scared or frightened? & 1 & 2 & 3 & 4 & 5 \\
\hline (12)Did you experience distention in the underarm or breast? & 1 & 2 & 3 & 4 & 5 \\
\hline (13)Did you feel chest or abdominal stuffiness? & 1 & 2 & 3 & 4 & 5 \\
\hline (14)Did you sigh without reason? & 1 & 2 & 3 & 4 & 5 \\
\hline (15)Did your body feel heavy or lethargic? & 1 & 2 & 3 & 4 & 5 \\
\hline (16)Did the palms of your hands or soles of your feet feel hot? & 1 & 2 & 3 & 4 & 5 \\
\hline (17)Did your hands or feet feel cold or clammy? & 1 & 2 & 3 & 4 & 5 \\
\hline (18)Did you feel cold easily in your abdomen, back, lower back or knees? & 1 & 2 & 3 & 4 & 5 \\
\hline (19)Were you sensitive to cold and tended to wear more clothes than others? & 1 & 2 & 3 & 4 & 5 \\
\hline (20)Did your body and face feel hot? & 1 & 2 & 3 & 4 & 5 \\
\hline $\begin{array}{l}\text { (21)Did you feel more vulnerable to cold than others (winter coldness, air conditioners, fans, } \\
\text { etc.)? }\end{array}$ & 1 & 2 & 3 & 4 & 5 \\
\hline (22)Did you catch colds more easily than others? & 1 & 2 & 3 & 4 & 5 \\
\hline (23)Did you sneeze even when you did not have a cold? & 1 & 2 & 3 & 4 & 5 \\
\hline (24)Did you have a runny or stuffy nose even when you did not have a cold? & 1 & 2 & 3 & 4 & 5 \\
\hline (25)Did you cough due to seasonal changes, temperature changes, or unpleasant odors? & 1 & 2 & 3 & 4 & 5 \\
\hline (26)Did you sweat easily when your physical activity increased slightly? & 1 & 2 & 3 & 4 & 5 \\
\hline (27)Did you forget things easily? & 1 & 2 & 3 & 4 & 5 \\
\hline (28)Did you have an excessively oily forehead and/or T-zone? & 1 & 2 & 3 & 4 & 5 \\
\hline (29)Were your lips redder than in the past? & 1 & 2 & 3 & 4 & 5 \\
\hline $\begin{array}{l}\text { (30)Did you have allergies? (e. g. medicine, food, odors, pollen, pet dander, or during seasonal } \\
\text { or weather change etc.) }\end{array}$ & 1 & 2 & 3 & 4 & 5 \\
\hline (31)Did you get hives/urticaria easily? & 1 & 2 & 3 & 4 & 5 \\
\hline (32)Did your skin have purpura (purple spots, ecchymosis) due to allergies? & 1 & 2 & 3 & 4 & 5 \\
\hline (33)Did black or purple bruises appear on your skin for no reason? & 1 & 2 & 3 & 4 & 5 \\
\hline (34)Did your skin turn red and show traces when you scratched it? & 1 & 2 & 3 & 4 & 5 \\
\hline (35)Did your skin or lips feel dry? & 1 & 2 & 3 & 4 & 5 \\
\hline (36)Did you have visible capillary (thread) veins on your cheeks? & 1 & 2 & 3 & 4 & 5 \\
\hline (37)Did you feel pain somewhere in your body? & 1 & 2 & 3 & 4 & 5 \\
\hline (38)Did you experience hot flashes? & 1 & 2 & 3 & 4 & 5 \\
\hline (39)Did your nose or your face feel greasy, oily, or shiny? & 1 & 2 & 3 & 4 & 5 \\
\hline (40)Did you have a dark face or get brown spots easily? & 1 & 2 & 3 & 4 & 5 \\
\hline (41)Did you get acne or sores easily? & 1 & 2 & 3 & 4 & 5 \\
\hline (42)Did you have upper eyelid swelling? & 1 & 2 & 3 & 4 & 5 \\
\hline (43)Did you get dark circles under the eyes easily? & 1 & 2 & 3 & 4 & 5 \\
\hline (44)Did your eyes feel dry and you used eye drops? & 1 & 2 & 3 & 4 & 5 \\
\hline (45)Were your lips darker, more blue or purple than usual? & 1 & 2 & 3 & 4 & 5 \\
\hline (46)Did you often feel parched and need to drink water? & 1 & 2 & 3 & 4 & 5 \\
\hline $\begin{array}{l}\text { (47)Did your throat feel strange (i.e., as if something was stuck or there was a lump in your } \\
\text { throat)? }\end{array}$ & 1 & 2 & 3 & 4 & 5 \\
\hline (48)Did you have a bitter or strange taste in your mouth? & 1 & 2 & 3 & 4 & 5 \\
\hline (49)Did your mouth feel sticky? & 1 & 2 & 3 & 4 & 5 \\
\hline (50)Was your abdomen flabby? & 1 & 2 & 3 & 4 & 5 \\
\hline (51)Did you have an abundance of phlegm, especially in your throat? & 1 & 2 & 3 & 4 & 5 \\
\hline $\begin{array}{l}\text { (52)Did you feel uncomforTable when you drank or ate something cold, or did you avoid to } \\
\text { drinking or eating cold items? }\end{array}$ & 1 & 2 & 3 & 4 & 5 \\
\hline (53)Could you adapt yourself to external natural or social environment changes? & 1 & 2 & 3 & 4 & 5 \\
\hline (54)Did you easily experience insomnia? & 1 & 2 & 3 & 4 & 5 \\
\hline $\begin{array}{l}\text { (55)Did you easily contract diarrhea when you were exposed to cold or ate (or drank) } \\
\text { something cold? }\end{array}$ & 1 & 2 & 3 & 4 & 5 \\
\hline (56)Did you pass sticky stools and/or feel that your bowel movement was incomplete? & 1 & 2 & 3 & 4 & 5 \\
\hline (57)Did you get constipated easily or have dry stools? & 1 & 2 & 3 & 4 & 5 \\
\hline (58)Did your tongue have a thick coating? & 1 & 2 & 3 & 4 & 5 \\
\hline (59)Did your urethral canal feel hot when you urinated, or did your urine have a dark color? & 1 & 2 & 3 & 4 & 5 \\
\hline (60)Was your vaginal discharge yellowish (only for female interviewees)? & 1 & 2 & 3 & 4 & 5 \\
\hline (60)Was your scrotum always wet (only for male interviewees)? & 1 & 2 & 3 & 4 & 5 \\
\hline
\end{tabular}




\section{Cellular Physiology Cell Physiol Biochem 2018;49:2088-2098

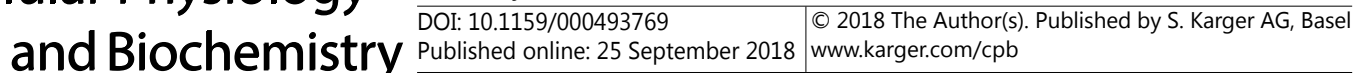

Table 2. Diagnostic standards for Yang deficiency, Yin deficiency, and Balanced constitutions

\begin{tabular}{lccc}
\hline & Yang deficiency & Yin deficiency & Balanced \\
\hline \multirow{2}{*}{ Main characteristics } & Cold intolerance & Heat intolerance & Energetic \\
& Cold hands, feet, stomach, and waist & Hot body, and face and eyes feel dry & Without any symptoms or characteristics of other constitutions \\
& Prefer hot food and drinks & Prefer cold food and drinks & \\
Susceptible to cold & Susceptible to heat & Sleep well \\
Secondary characteristics & Watery stool & Constipated & Cold tolerance \\
& Whitish skin & Emaciation & \\
& Nocturia & Feod memory & \\
& Tender and pale tongue & Red lips and dry skin & \\
& & Dry and reed tongue & \\
\hline
\end{tabular}

group. Subjects were Chinese Han individuals, aged 20-50 years old, from both genders. Three groups of subjects with no significant difference in age $(F=0.126, P$-value $=0.882)$ and gender $(P$-value $=0.5)$ were chosen (Table 3 , Table 4$)$. Fisher's Exact Test was used for gender analysis due to the small group size. This research project was approved by the local ethics committee of Beijing University of Chinese Medicine (2012BZHYLL0301), and all subjects were informed and consented to this study.

Saliva samples of subjects were collected by buccal swabbing from the clean oral cavity of 15 subjects. The samples were frozen immediately in liquid nitrogen and stored at $-80^{\circ} \mathrm{C}$. Subjects were told to abstain from drinking, eating, smoking and exercising two hours prior to sample collection.

\section{Extraction of Total Saliva RNA}

Total-RNA extraction was performed with TRIzol (Invitrogen, Carlsbad, CA, USA) according to the manufacturer's instructions. RNA sample quality was determined using NanoDrop®ND-1000 (Nanodrop, Thermo Scientific, MA, USA). To remove any genomic DNA contamination, the samples were treated with DNase (DNAfree kit, Ambion, Austin, TX, USA). All extracted RNAs passed the quality inspection.
Table 3. Age composition of Yang deficiency, Yin deficiency, and Balanced constitutions

\begin{tabular}{lc}
\hline group & Age(years) \\
\hline Balanced & $31.6 \pm 8.0$ \\
Yang deficiency & $29.6 \pm 10.3$ \\
Yin deficiency & $32.2 \pm 7.1$ \\
F-value & 0.126 \\
P-value & 0.882 \\
\hline
\end{tabular}

Table 4. Gender composition of Yang deficiency, Yin deficiency, and Balanced constitutions. Note: Fisher's Exact Test was used in Table 3. due to the small group size

\begin{tabular}{lcccc}
\hline Group & Male & Female & $\chi^{2}$ & P \\
\hline Balanced & 3 & 2 & 2.4 & 0.5 \\
Yang deficiency & 1 & 4 & & \\
Yin deficiency & 1 & 4 & & \\
\hline
\end{tabular}

\section{miRNA Chip Analysis}

The samples were then analyzed with Human miRNA OneArray®v7 (Phalanx Biotech Group, Hsinchu, China Taiwan). The microarrays are made of polydeoxynucleotide probes spotted onto a proprietary chemical layer coated on top of a 1"x3"standard format microarray glass slide. Each probe is spotted onto the array in a highly consistent manner using proprietary, non-contact spotting technology. Each microarray contains 2548 unique human miRNA probes and 124 experimental control probes. Each unique probe has 3 features, and probes contain $100 \%$ of Sanger miRBasev21 miRNA content. Targets were labeled using ULS miRNA Labeling Kit (Kreatech Diagnostics, Amsterdam, Netherlands). Hybridization process was completed using miRNA OneArray Hybridization Buffer v3 and miRNA OneArray Hybridization Buffer II. The miRNA chip was scanned by GenePix 4000B Microarray Scanner (Axon Instruments Inc, Union, CA, USA) and was then analyzed by GenePix 4.1 software.

\section{miRNA target gene prediction}

Target genes of differentially expressed miRNAs were predicted using DIANA-TarBasev7.0, which aims to provide, for the first time, hundreds of thousands of high-quality, manually, experimentally validated miRNA-gene interactions, enhanced with detailed meta-data [25].

\section{Pathway analysis of related target genes}

The combined effect of differentially expressed miRNAs on pathways was determined based on the related target genes using KEGG analysis of DIANA-mirPathv.3. The threshold of significance of pathways was defined by $P$-value and false discovery rate (FDR). The selection criterion of significant KEGG pathways was $P$-value $<0.01$. 


\section{Cellular Physiology Cell Physiol Biochem 2018;49:2088-2098 \begin{tabular}{ll|l} 
and BiOChemistry & DOI: 10.1159/000493769 & $\begin{array}{l}\text { C } 2018 \text { The Author(s). Published by S. Karger AG, Basel } \\
\text { www.karger.com/cpb }\end{array}$ \\
\hline
\end{tabular}

\section{Results}

\section{Cluster analysis and Principal Component Analysis}

To investigate objective classification of Yang deficiency and Yin deficiency constitution, saliva from the 15 health subjects was collected, and miRNA expression profile was determined using the Human miRNA OneArray ${ }^{\circledR}$ v7. We carried out unsupervised clustering analysis using all expressed miRNAs to determine whether expression profile could be used to classify constitutional individuals. Samples were clustered into three distinct groups, largely consistent with the Yang deficiency, Yin deficiency and Balanced constitutions (Fig. 1A).

Principal component analysis yielded similar results (Fig. 1B).This finding demonstrated that miRNA expression profile-based clustering of subjects largely overlapped with the TCM classification.

\section{Differentially expressed miRNAs}

The standardized questionnaire method for classifying the TCM constitution is subjective and cases of exaggeration or understatement of symptoms cannot be avoided. Thus, we selected the overlapping samples from standardized questionnaire judgment and miRNA expression clustering for a more accurate research. We excluded one sample in Balanced constitution, namely P-1(male, 36 years old). A total of 14 samples were included in the final analysis. Differentially expressed miRNAs were screen based on 1.5 Fold change, means $\log _{2} \mid$ Ratio $\mid \geq 0.585$ and $P$-value $<0.05$. A total of 81 specific miRNAs were screened in Yang deficiency constitution, out of which 33 were up-regulated (Fig. 2A) and 48 were down-regulated (Fig. 2B). Of these, hsa-miR-4443 was the most significantly up-regulated miRNA and hsa-miR-2681-3p was the most significantly down-regulated miRNA. A total

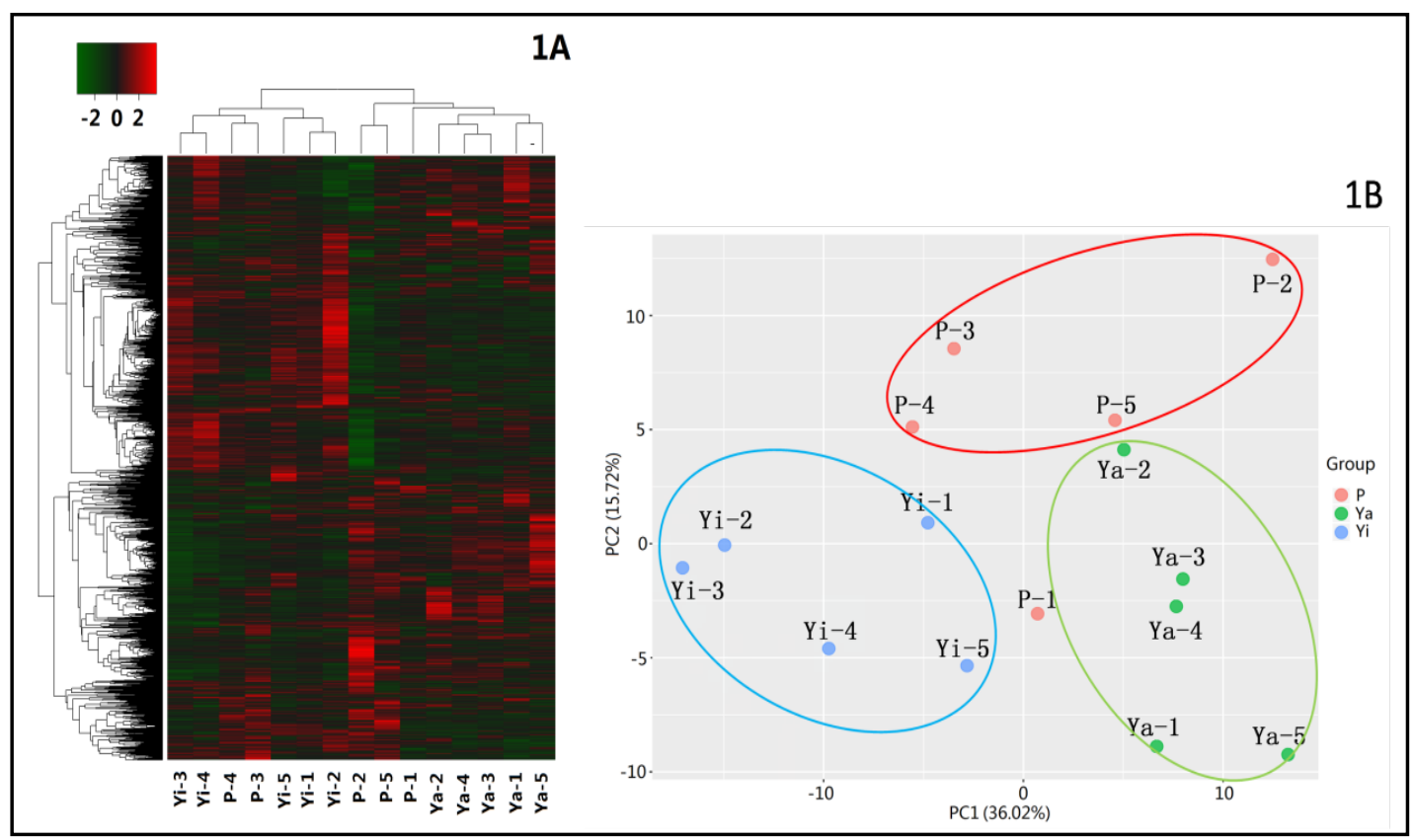

Fig. 1. Classification of constitutional subjects based on miRNA-expression profiles. (A) Heatmap showing the hierarchical cluster analysis of miRNA-expression profiles in saliva obtained from the subjects with Yang deficiency or Yin deficiency or Balanced constitutions. The scale bar indicates the level of miRNA expression: red, high level of expression; green, low level of expression. (B) Principal component analysis (PCA) was conducted using the same miRNA set. Circles represent individual subjects, and colors within circles indicate the constitutional group: blue, Yin deficiency constitution; green, Yang deficiency constitution; red, Balanced constitution. 


\section{Cellular Physiology Cell Physiol Biochem 2018;49:2088-2098 and Biochemistry Published online:25 September 2018 年 $\begin{aligned} & \text { O } 2018 \text { The Author(s). Published by S. Karger AG, Basel } \\ & \text { wwrger.com/cpb }\end{aligned}$

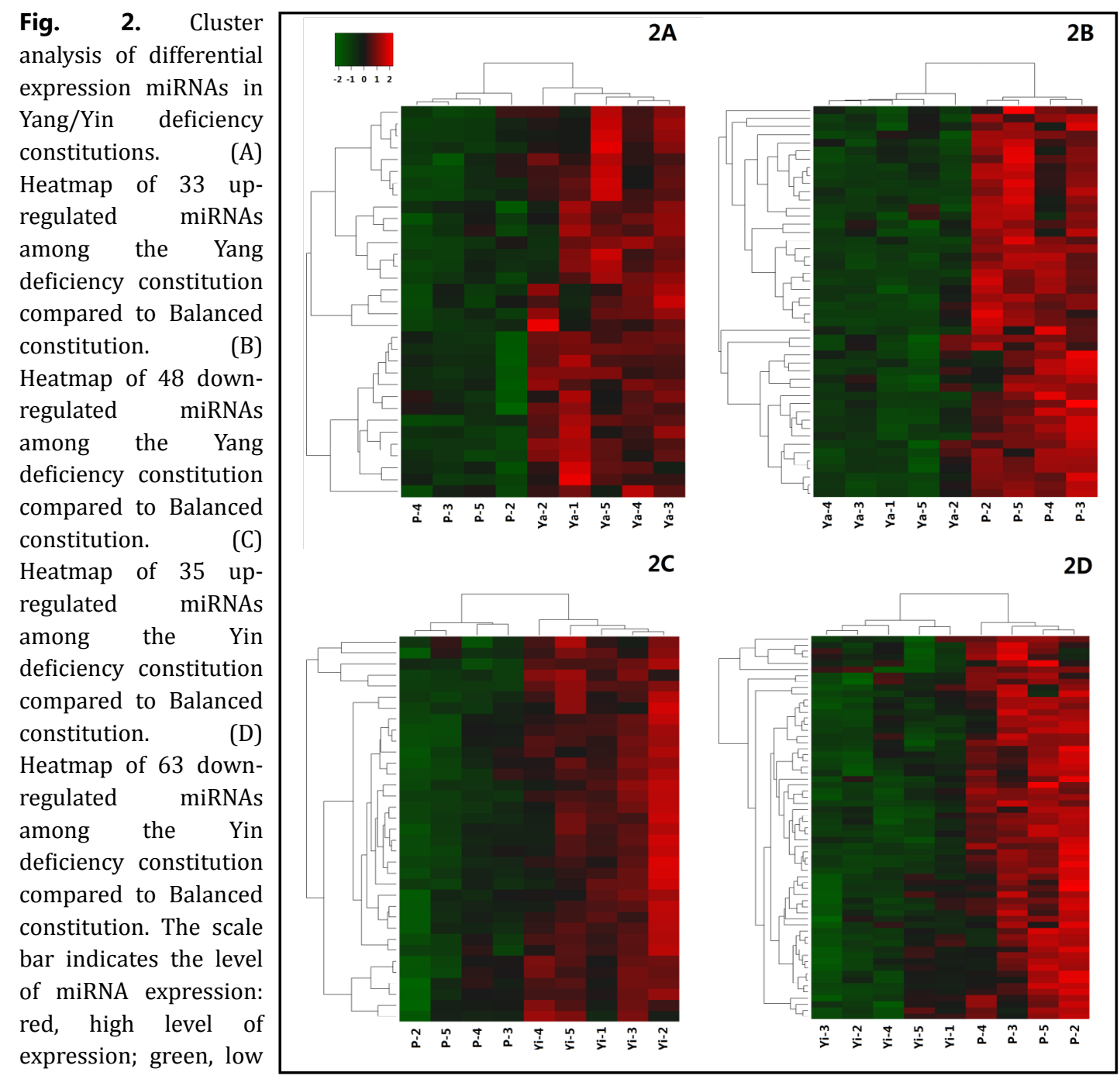

level of expression.

of 98 specific miRNAs were screened in Yin deficiency constitution, out of which 35 were up-regulated (Fig. 2C) and 63 were down-regulated (Fig. 2D). Of these, hsa-miR-4455 was the most significantly up-regulated miRNA and hsa-miR-1343-3p was the most significantly down-regulated miRNA. The degree of differentially expressed miRNAs has been listed (Table 5, Table 6). Further comparative analysis found that 16 identical miRNAs existed in both Yang and Yin deficiency constitution, out of which 1 was up-regulated and 15 were downregulated. As a result, 65 unique miRNAs were observed in Yang deficiency constitution and 82 unique miRNAs were observed in Yin deficiency constitution.

\section{Prediction of miRNA-regulated Target Genes}

As a gene regulator, combinatorial regulation is an important feature for miRNA. Usually, a given miRNA has multiple different mRNA targets, and multiple miRNAs might target one gene [26]. In our study, using the DIANA-TarBasev7.0, miRNA-regulated target genes were predicted. A total of 11, 409 target genes were predicted by 81 differential miRNAs in Yang deficiency constitution and a total of 12, 151 target genes were predicted by 98 differential miRNAs in Yin deficiency constitution. Meanwhile, a total of 10, 314 target genes were predicted by 65 unique miRNAs in Yang deficiency constitution and a total of 11, 270 target genes were predicted by 82 unique miRNAs in Yin deficiency constitution. The target genes which showed significant differences $(P<0.05)$ were selected.

\section{KARGER}


Table 5. The degree of all differentially expressed miRNAs in Yang deficiency constitution compared to Balanced constitution

\begin{tabular}{|c|c|c|c|c|c|c|c|}
\hline Up-regulated & $\begin{array}{c}\text { Log2 } \\
\text { (ratio) }\end{array}$ & Up-regulated & $\begin{array}{c}\text { Log2 } \\
\text { (ratio) }\end{array}$ & Down-regulated & $\begin{array}{c}\text { Log2 } \\
\text { (ratio) }\end{array}$ & Down-regulated & $\begin{array}{c}\text { Log2 } \\
\text { (ratio) }\end{array}$ \\
\hline hsa-miR-23a-5p & 1.01 & & & hsa-miR-516b-5p & -1.36 & hsa-miR-3664-3p & -0.59 \\
\hline hsa-miR-769-3p & 0.76 & & & hsa-let-7c-5p & -0.64 & hsa-miR-4419b & -0.83 \\
\hline hsa-miR-584-5p & 0.65 & & & hsa-let-7a-5p & -0.76 & hsa-miR-2681-3p & -2.79 \\
\hline hsa-miR-125a-3p & 0.71 & & & hsa-miR-205-5p & -1.10 & hsa-miR-6873-5p & -1.36 \\
\hline hsa-miR-542-5p & 0.78 & & & hsa-miR-1246 & -1.42 & hsa-miR-7159-5p & -0.93 \\
\hline hsa-miR-4323 & 0.66 & & & hsa-miR-922 & -0.64 & hsa-miR-6732-5p & -0.70 \\
\hline hsa-miR-3648 & 0.67 & & & hsa-miR-524-5p & -1.20 & hsa-miR-7111-5p & -1.15 \\
\hline hsa-miR-3972 & 0.81 & & & hsa-miR-27b-3p & -0.93 & hsa-miR-7113-5p & -0.88 \\
\hline hsa-miR-4665-5p & 1.00 & $6782-3 n$ & 062 & hsa-miR-27a-3p & -0.77 & hsa-miR-6755-5p & -0.81 \\
\hline hsa-miR-3162-3p & 0.63 & $782-3 n$ & $\begin{array}{l}0.62 \\
0.70\end{array}$ & hsa-miR-23a-3p & -1.34 & hsa-miR-6746-5p & -0.96 \\
\hline hsa-miR-3925-5p & 0.73 & h & $\begin{array}{l}0.10 \\
0.72\end{array}$ & hsa-miR-23b-3p & -1.25 & hsa-miR-6767-5p & -0.67 \\
\hline hsa-miR-4443 & 2.20 & $\mathrm{~h}$ & $\begin{array}{l}0.72 \\
0.72\end{array}$ & hsa-miR-202-3p & -0.59 & hsa-miR-6738-5p & -1.56 \\
\hline hsa-miR-4447 & 0.87 & $\mathrm{p}$ & 0.72 & hsa-miR-3121-3p & -0.73 & hsa-miR-6778-5p & -0.65 \\
\hline hsa-miR-4472 & 0.89 & 5884- & $\begin{array}{l}0.99 \\
0.96\end{array}$ & hsa-miR-3152-3p & -0.70 & hsa-miR-6895-5p & -1.04 \\
\hline hsa-miR-4497 & 0.86 & $-m$ & 0.90 & hsa-miR-1973 & -0.91 & hsa-miR-6794-5p & -1.03 \\
\hline hsa-miR-4505 & 0.60 & $-m$ & $\begin{array}{l}0.74 \\
0.67\end{array}$ & hsa-miR-3153 & -1.21 & hsa-miR-6801-5p & -0.82 \\
\hline hsa-miR-4525 & 1.28 & 列 & 0.67 & hsa-miR-3162-5p & -0.90 & hsa-miR-6795-5p & -0.74 \\
\hline hsa-miR-4747-5p & 1.16 & & & hsa-miR-4253 & -1.74 & hsa-miR-7154-3p & -0.60 \\
\hline hsa-miR-3620-5p & 0.59 & & & hsa-miR-4271 & -1.14 & hsa-miR-6777-5p & -0.89 \\
\hline hsa-miR-6132 & 1.19 & & & hsa-miR-4688 & -0.88 & hsa-miR-605-3p & -0.63 \\
\hline hsa-miR-6845-5p & 0.69 & & & hsa-miR-4728-5p & -0.90 & hsa-miR-1910-3p & -1.01 \\
\hline hsa-miR-6864-5p & 0.96 & & & hsa-miR-4687-3p & -0.82 & hsa-miR-504-3p & -0.60 \\
\hline hsa-miR-6813-5p & 0.91 & & & hsa-miR-3605-5p & -0.59 & hsa-miR-6787-5p & -1.06 \\
\hline hsa-miR-6880-3p & 0.88 & & & hsa-miR-4436b-3p & -0.73 & hsa-miR-4713-3p & -1.93 \\
\hline
\end{tabular}

Analysis of signaling pathways regulated by unique miRNAs To further explore the characteristic biological functions of the differentially expressed unique miRNAs, signaling pathways $(P<0.01)$ were analyzed using DIANA-mirPathv.3 (Fig. 3). The pathway analysis between Yang deficiency constitution and Balanced constitution showed that the target genes regulated by the 65 unique miRNAs are involved in a total of 27 signaling pathways (Fig. 3A). The analysis Table 6. The degree of all differentially expressed miRNAs in Yin deficiency constitution compared to Balanced constitution

\begin{tabular}{|c|c|c|c|c|c|c|c|}
\hline Up-regulated & $\begin{array}{c}\log 2 \\
\text { (ratio) }\end{array}$ & Up-regulated & $\begin{array}{c}\text { Log2 } \\
\text { (ratio) }\end{array}$ & Down-regulated & $\begin{array}{c}\text { Log2 } \\
\text { (ratio) }\end{array}$ & Down-regulated & $\begin{array}{c}\text { Log2 } \\
\text { (ratio) }\end{array}$ \\
\hline hsa-miR-1289 & 0.69 & & & hsa-miR-498 & -1.26 & & \\
\hline hsa-miR-1265 & 0.60 & & & hsa-miR-516b-5p & -0.74 & hsa-miR-4689 & -0.91 \\
\hline hsa-miR-181b-5p & 0.74 & & & hsa-miR-205-5p & -1.08 & miR-4697-5p & -1.07 \\
\hline hsa-miR-1-3p & 0.88 & & & hsa-miR-302c-5p & -1.03 & hsa-miR-4706 & -0.62 \\
\hline hsa-miR-122-5p & 1.01 & & & hsa-miR-638 & -0.64 & hsa-miR-4725-3p & -0.69 \\
\hline hsa-miR-493-3p & 0.60 & & & hsa-miR-203a-3p & $\begin{array}{l}-0.04 \\
-0.78\end{array}$ & hsa-miR-3936 & -0.77 \\
\hline hsa-miR-138-5p & 0.81 & & & hsa-miR-936 & -0.60 & hsa-miR-4433a-3p & -0.60 \\
\hline hsa-miR-99b-3p & 0.82 & & & hsa-miR-150-3p & $\begin{array}{l}-0.00 \\
-0.67\end{array}$ & hsa-miR-4444 & -1.09 \\
\hline hsa-miR-330-5p & 0.59 & & & hsa-miR-198 & -0.81 & hsa-miR-4458 & -0.82 \\
\hline hsa-miR-1272 & 0.63 & & & hsa-miR-1287-5p & -0.94 & hsa-miR-4652-5p & -0.73 \\
\hline hsa-miR-2861 & 0.64 & & & hsa-miR-524-5p & -0.92 & hsa-miR-345-3p & -0.81 \\
\hline hsa-miR-4273 & 0.59 & & & hsa-miR-23a-3p & $\begin{array}{l}-0.92 \\
-0.83\end{array}$ & hsa-miR-5195-3p & -1.26 \\
\hline hsa-miR-205-3p & 0.81 & & & hsa-miR-23b-3p & $\begin{array}{l}-0.83 \\
-0.83 \quad r\end{array}$ & hsa-miR-5010-5p & -1.03 \\
\hline hsa-miR-3198 & 1.09 & & & hsa-miR-551b-5p & $\begin{array}{l}-0.83 \\
-0.64\end{array}$ & hsa-miR-6716-5p & -1.06 \\
\hline hsa-miR-4498 & 0.66 & & & hsa-miR-5 & & hsa-miR-6511b-5p & -0.69 \\
\hline hsa-miR-1587 & 0.64 & hsa-miR-6862-3p & 0.65 & hsa-miR-185-5p & $\begin{array}{l}-1.14 \\
-0.83\end{array}$ & hsa-miR-6718-5p & -0.77 \\
\hline hsa-miR-4502 & 0.79 & hsa-miR-329-5p & 0.95 & & & hsa-miR-6076 & -0.98 \\
\hline hsa-miR-4537 & 0.60 & hsa-miR-153-5p & 0.92 & hsa-miR-1914-3p & $\begin{array}{l}-0.01 \\
-0.99\end{array}$ & hsa-miR-6507-5p & -0.79 \\
\hline hsa-miR-4538 & 0.60 & & & hsa-miR-3179 & -0.69 & hsa-miR-6826-5p & -0.71 \\
\hline hsa-miR-4675 & 0.69 & & & hsa-miR-4294 & -0.59 & hsa-miR-6873-5p & -1.14 \\
\hline hsa-miR-4716-5p & 0.64 & & & hsa-miR-3122 & -0.69 & hsa-miR-7111-5p & -0.90 \\
\hline hsa-miR-4761-5p & 0.60 & & & hsa-miR-3124-5p & -1.18 & hsa-miR-6755-5p & -0.68 \\
\hline hsa-miR-4474-3p & 0.62 & & & hsa-miR-3153 & -1.26 & hsa-miR-6774-5p & -0.67 \\
\hline hsa-miR-4455 & 1.31 & & & hsa-miR-3156-5p & -0.64 & hsa-miR-6738-5p & -0.75 \\
\hline hsa-miR-4711-3p & 0.63 & & & hsa-miR-3 & $\begin{array}{l}-0.98 \\
-0.98\end{array}$ & hsa-miR-6781-5p & -1.13 \\
\hline hsa-miR-4671-3p & 0.66 & & & hsa-miR-3176 & -0.78 & hsa-miR-6804-5p & -0.60 \\
\hline hsa-miR-4999-5p & 1.01 & & & hsa-miR & -1.03 & hsa-miR-6802-5p & -0.98 \\
\hline hsa-miR-8086 & 0.62 & & & hsa-miR-514b-5p & $\begin{array}{l}-1.05 \\
-0.79\end{array}$ & hsa-miR-6815-5p & -0.84 \\
\hline hsa-miR-6813-5p & 0.64 & & & hsa-miR-4257 & -0.63 & $85-5 p$ & -1.21 \\
\hline hsa-miR-6843-3p & 0.65 & & & hsa-miR-4253 & $\begin{array}{l}-0.05 \\
-1.33\end{array}$ & hsa-miR-504-3p & -0.61 \\
\hline $6739-3 p$ & 0.62 & & & hsa-miR-4271 & -0.82 & $-5 p$ & -1.17 \\
\hline hsa-miR-6822-3p & 0.59 & & & hsa-miR-3714 & -0.59 & hsa-miR-1343-3p & -1.35 \\
\hline
\end{tabular}

between Yin deficiency constitution and Balanced constitution showed that the target genes regulated by the 82 unique miRNAs are involved in a total of 20 significant pathways (Fig. 3B). These pathways were classified into functional groups according to KEGG pathway database. These functional groups were mainly distributed in human diseases, endocrine system, metabolism, nervous system, signal transduction. A total of 11 pathways, including thyroid hormone signaling pathway, oxytocin signaling pathway, proteoglycans in cancer, morphine addiction, lysine degradation, long-term depression, glutamatergic synapse, hippo 


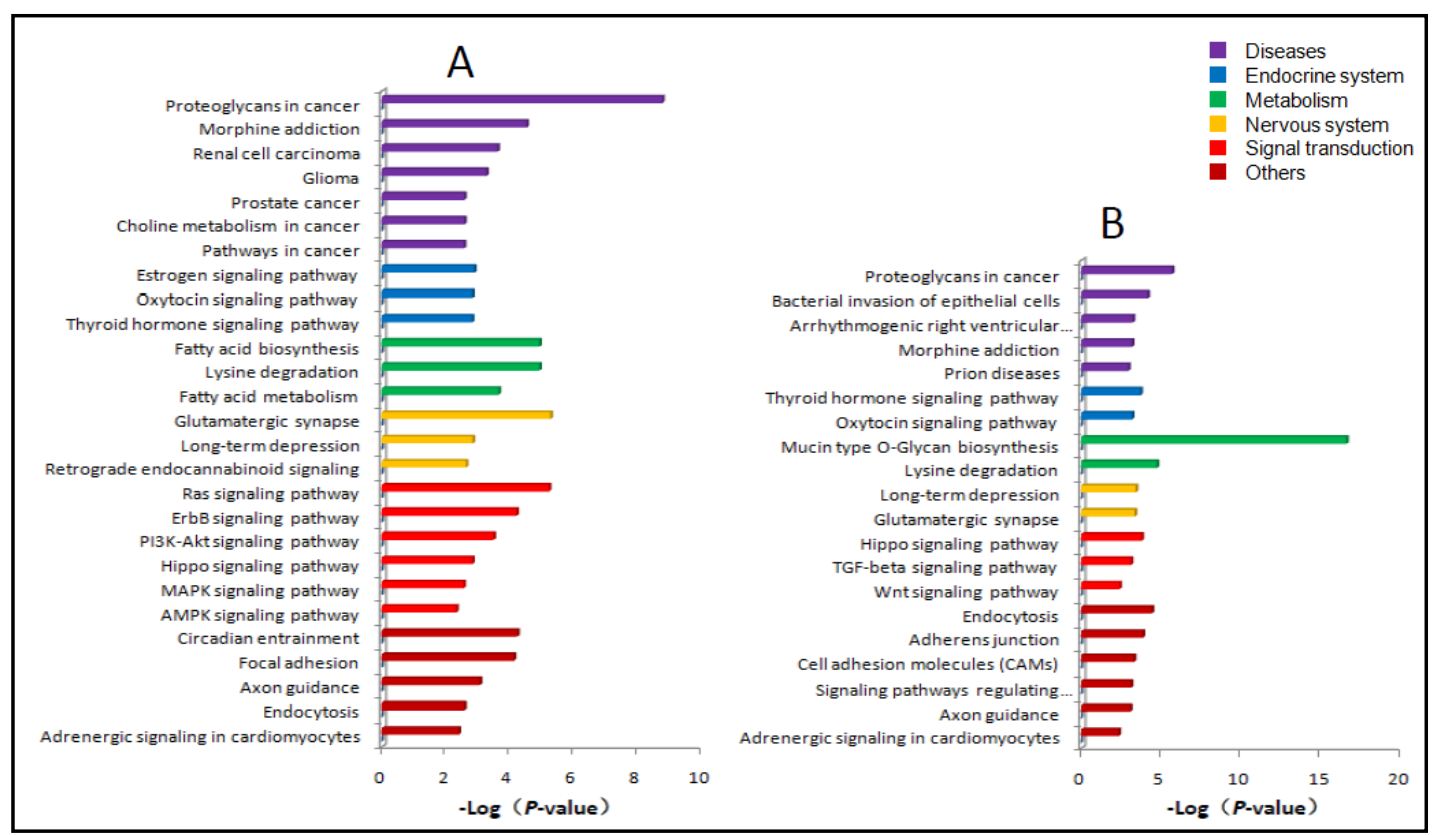

Fig. 3. Signaling pathways analysis based on target genes of unique miRNAs. (A) Enriched pathways based on target genes of 65 unique miRNAs of Yang deficiency compared to Balanced constitution. (B) Enriched pathways based on target genes of 82 unique miRNAs of Yin deficiency compared to Balanced constitution. The vertical axis is the pathway category and the horizontal axis is the - $\log \mathrm{P}$ of pathway, $\log \mathrm{P}$ is the logarithm of $\mathrm{P}$-value, and $\mathrm{P}<0.01$ is considered significant.

signaling pathway, endocytosis, axon guidance, adrenergic signaling in cardiomyocytes, were enriched in both Yang deficiency and Yin deficiency constitution. The results show that these two constitutions may have similar abnormalities in some aspect, but the respective manifestations may be different. Among them, the abnormality of thyroid hormones may be related to both cold and heat intolerance. Thyroid hormones maintain the body's functional activity and have a critical role in thermogenesis. Based on the critical role of thyroid hormone in thermogenesis and the role of miRNA, these unique miRNAs that regulated target genes of the thyroid hormone signaling pathway may lead to cold intolerance or heat intolerance. Further analysis of the regulatory networks could reveal the relationship between miRNAs and target genes in thyroid hormone signaling pathway. We found that hsa-miR-27b-3p, hsa-miR-27a-3p, hsa-miR-3121-3p regulate target genes in Yang deficiency constitution to a higher degree (Fig. S1A - For all supplemental material see www.karger. com/10.1159/000493769/). Meanwhile, hsa-miR-153-5p, hsa-miR-330-5p, hsa-miR-498, hsa-miR-302c-5p, hsa-miR-185-5p regulate target genes in Yin deficiency constitution to a higher degree (Fig. S1B).

\section{Discussion}

Based on TCM theory, there are nine constitutions in the Chinese Han population, namely, a balanced constitution and eight unbalanced constitutions [4]. Unbalanced constitutions include Qi deficiency, Yang or Yin deficiency, Phlegm-dampness, Damp-heat, Blood stasis, Qi stagnation, and Inherited special constitutions. These unbalanced constitutions are considered to be related to diseases or are the risk factors of diseases. As a result, the research on TCM constitution has important clinical significance in the prediction and prevention of diseases. However, the clinical roles of TCM constitution should be based on accurate and objective classification. As a class of regulatory, small, non-coding RNA, miRNAs can regulate post-transcriptional gene expression by pairing to complementary binding sites within 


\section{Cellular Physiology Cell Physiol Biochem 2018;49:2088-2098 \\ and Biochemistry \begin{tabular}{l|l} 
DOI: 10.1159/000493769 & $\begin{array}{l}\text { O 2018 The Author(s). Published by S. Karger AG, Basel } \\
\text { www.karger.com/cpb }\end{array}$
\end{tabular} \\ Chen et al.: mRNA Expression Profile of Saliva in Yang/Yin Deficiency Constitution}

the 3'untranslated region of hundreds of target mRNAs [27]. The role of miRNAs as key regulators of a wide variety of fundamental cellular processes is increasingly recognized in many aspects of biology and biomedicine [28]. miRNA regulation may be intricately related to distinctive features of TCM constitution and may contribute to its objective classification. However, few studies have been conducted in this field.

Yang deficiency and Yin deficiency constitution account for $9.04 \%$ and $8.27 \%$ of the distribution of nine constitutions, respectively [29]. They exhibit differences in biological features and disease risks. Our team has researched on the metabolomics and global gene expression profiles and found clues that contribute to the classification [7, 9]. We hope to enrich the evidence for objective classification from the level of post-transcriptional gene regulation through the analysis of miRNAs in saliva. In our study, miRNA expression profile of saliva was constructed and systematic difference in miRNA expression profile was exhibited by high-throughput gene chip technology. The systematic difference indicated that miRNAs play a potential role in the classification of these two constitutions. In addition, prediction of target genes regulated by differentially expressed unique miRNAs and enrichment analysis of signaling pathways were implemented to further explore the characteristic biological functions. The results show that the abnormalities in signal transduction, metabolism and the endocrine and nervous systems may exist in both Yang deficiency and Yin deficiency constitution. Multiple cancers could be related to Yang deficiency constitution, while cardiovascular diseases, infectious diseases and certain cancers could be related to Yin deficiency constitution. In the condition of health, these two constitutions have shown abnormalities in physiological function and susceptibility to disease.

Cold intolerance is the main characteristic of Yang deficiency constitution and heat intolerance is the main characteristic of Yin deficiency constitution [4]. The occurrence of cold intolerance or heat intolerance is a very complicated process in which abnormal thermogenesis may be a factor. Thyroid hormones have extensive physiological functions, one of which is to regulate thermogenesis, and that includes both obligatory and facultative thermogenesis [30, 31]. Therefore, thyroid hormone abnormalities may be associated with the main characteristics of Yang deficiency or Yin deficiency constitution. Previous studies have also provided some evidence. A study of endocrine function found that Yang deficiency constitution may be related to the hypo-function of hypothalamic-pituitary-thyroid axis [6]. Another study about molecular basis of cold intolerance in Yang deficiency constitution found that impaired thermogenesis could be related to the abnormal expression of thyroid hormone receptor beta (TR $\beta$ ) in peripheral white blood cells [32]. In our study the thyroid hormone signaling pathway was significantly enriched in both Yang deficiency and Yin deficiency constitution and was affected by the target genes that were regulated by the unique miRNAs. These unique miRNAs were completely different in Yang deficiency and Yin deficiency constitution. Therefore, there was different effect on thyroid hormone signaling pathway and consequently, thyroid hormones. Based on the different effect, abnormality of thermogenesis may exist in these two constitutions, but different manifestation may be observed. Further research found that target genes ATP1B1, ATP1B2, ATP1B3, ATP1B4, ATP1A2, ATP1A3 and ATP1A4 exist in the thyroid hormone signaling pathway of Yang deficiency constitution. At the same time, the target genes ATP1B1, ATP1B2, ATP1B4, ATP1A2 and ATP1A3 exist in the thyroid hormone signaling pathway of Yin deficiency constitution. These target genes were all related to $\mathrm{Na}^{+} / \mathrm{K}^{+}$-ATPase. Thyroid hormone may promote thermogenesis by stimulating the activity and quantity of $\mathrm{Na}^{+} / \mathrm{K}^{+}$-ATPase [33]. Therefore, abnormal expression of these target genes may be more important for cold intolerance or heat intolerance. As a result, the differentially expressed unique miRNAs that regulate these target genes may be the main factors that result in cold intolerance or heat intolerance. 


\section{Cellular Physiology Cell Physiol Biochem 2018;49:2088-2098 \begin{tabular}{ll|l} 
and Biochemistry Published online: 25 September 2018 & $\begin{array}{l}\text { (c) } 2018 \text { The Author(s). Published by S. Karger AG, Basel } \\
\text { www.karger.com/cpb }\end{array}$ \\
\hline
\end{tabular}

\section{Conclusion}

In conclusion, by analyzing the difference of miRNA expression profile of saliva, we found that miRNAs play a role in objective classification of Yang deficiency and Yin deficiency constitution. The findings may further explain the characteristics and susceptibility to diseases of these two constitutions. As a relatively small number of subjects, mainly from Beijing, were enrolled in the current study, further investigations involving larger cohorts comprised of different geographic regions and the other unbalanced constitutions are needed to confirm our findings.

\section{Acknowledgements}

This study was supported by the National Key Basic Research Program of China $(973$ Program; No. 2011 CB505400).

\section{Disclosure Statement}

The authors declare no conflict of interests.

\section{References}

1 Auffray C, Charron D, Hood L: Predictive, preventive, personalized and participatory medicine: back to the future. Genome Med 2010;2:1-3.

2 Riddle MS, Murray JA, Porter CK: The incidence and risk of celiac disease in a healthy US adult population. Am J Gastroenterol 2012;107:1248-1255.

-3 Suh B, Shin DW, Kwon HM, Yun JM, Yang HK, Ahn E, Lee H, Park JH, Cho B: Elevated neutrophil to lymphocyte ratio and ischemic stroke risk in generally healthy adults. PLos One 2017;12:e0183706.

4 Wang Q: Classification and diagnosis basis of nine basic constitutions in Chinese medicine. J Beijing Univ Tradit Chin Med 2005;28:1-8.

$5 \quad$ Wang Q: On Chinese constitutional theory from three aspects. J Beijing Univ Tradit Chin Med 2008;31:653655.

6 Wang Q, Yao S, Dong J, Wu H, Wu C, Xia Z, Shi H, Pang G, Deng Q, Zhao J, Cai J, Cui Z: Changes of endocrine and immune function in subjects of yang deficiency constitution. J Chin Integr Med 2008;6:1226-1232.

$7 \quad$ Li Y, Wang Q, Yuan Z: NMR-based Metabonomics studies on Serum and Urine of Yang-deficiency Constitution. Chem J Chin Univ 2011;32:2521-2527.

8 Wu Y, Cun Y, Dong J, Shao J, Luo S, Nie S, Yu H, Zheng B, Wang Q, Xiao C: Polymorphisms in PPARD, PPARG and APM1 associated with four types of traditional Chinese medicine constitutions. J Genet Genomics 2010;37:371-379.

-9 Yu R, Liu D, Yang Y, Han Y, Li L, Zheng L, Wang J, Zhang Y, Li Y, Wang Q-F, Wang Q: Expression profilingbased clustering of healthy subjects recapitulates classifications defined by clinical observation in Chinese medicine. J Genet Genomics 2017;44:191-197.

-10 Kawahara R, Bollinger JG, Rivera C, Ribeiro AC, Brandao TB, Paes Leme AF, MacCoss MJ: A targeted proteomic strategy for the measurement of oral cancer candidate biomarkers in human saliva. Proteomic 2016;16:159-173.

11 Russo I, Saponeri A, Michelotto A, Alaibac M: Salivary sample for the diagnosis of pemphigus vulgaris using the BIOCHIP approach: a Pilot Study. Vivo 2017;31:97-99.

12 Bartel DP: MicroRNAs: genomics, biogenesis, mechanism, and function. Cell 2004;116:281-297.

-13 Bueno MJ, Pérez dCI, Malumbres M: Control of cell proliferation pathways by microRNAs. Cell Cycle 2008;7:3143-3148.

14 Kanwar JR, Mahidhara G, Kanwar RK: MicroRNA in human cancer and chronic inflammatory diseases. Front Biosci 2010;2:1113-1126. 


\section{Cellular Physiology Cell Physiol Biochem 2018:49:2088-2098

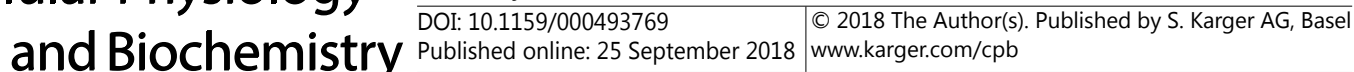

15 Sonntag KC: MicroRNAs and deregulated gene expression networks in neurodegeneration. Brain Res 2010;1338:48-57.

16 Megan B, Ashley S, Viravuth P: Dynamic microRNA-101a and Fosab expression controls zebrafish heart regeneration. Development 2015;142:4026-4037.

17 Li G, Shen Q Li C, Li D, Chen J, He M: Identification of circulating McroRNAs as novel potential biomarkers for hepatocellular carcinoma detection: a systematic review and meta-analysis. Clin Transl Oncol 2015;17:684-693.

18 Hou Y, Wang X, Chen Y, Mu S: MicroRNA-145 as ideal biomarker for the diagnosis of various carcinomas. Tumor Biol 2015;36:2641-2649.

19 Hackl M, Heilmeier U, Weilner S, Grillari J: Circulating microRNAs as novel biomarkers for bone diseasesComplex signatures for multifactorial diseases? Mol Cell Endocrinol 2016;432:83-95.

20 Park NJ, Zhou H, Elashoff D, Henson BS, Kastratovic DA, Abemayor E, Wong DT: Salivary microRNA: discovery, characterization, and clinical utility for oral cancer detection. Clin Cancer Res 2009;15:54735477.

-21 Hanke M, Hoefig K, Merz H, Feller AC, Kausch I, Jocham D, Warnecke JM, Sczakiel G: A robust methodology to study urine microRNA as tumor marker: microRNA-126 and microRNA-182 are related to urinary bladder cancer. Urol Oncol 2010;655-661.

22 Kosaka N, Izumi H, Sekine K, Ochiya T: microRNA as a new immune-regulatory agent in breast milk. Silence 2010;1:1-7.

-23 Skog J, Wurdinger T, Van SR, Meijer DH, Gainche L, Sena-Esteves M, Curry WT, Carter RS, Krichevsky AM, Breakefield XO: Glioblastoma microvesicles transport RNA and proteins that promote tumor growth and provide diagnostic biomarkers. Nat Cell Biol 2008;10:1470-1476.

24 Etheridge A, Lee I, Hood L, Galas D, Wang K: Extracellular microRNA: A new source of biomarkers. Mutat Res 2011;717:85-90.

25 Vlachos IS, Paraskevopoulou MD, Karagkouni D, Georgakilas G, Vergoulis T, Kanellos I, Anastasopoulos IL, Maniou S, Karathanou K, Kalfakakou D, Fevgas A, Dalamagas T, Hatzigeorgiou SG: DIANA-TarBase v7.0: indexing more than half a million experimentally supported miRNA: mRNA interactions. Nucleic Acids Res 2015;43:D153-159.

26 Krek A, Grün D, Poy MN, Wolf R, Rosenbery L, Epstein EJ, MacMenamin P, Piedade I, Gunsalus KC, Stoffel M, Rajewsky N: Combinatorial microRNA target predictions. Nature Genetics 2005;37:495-500.

27 Gottesman S: Small RNAs shed some light. Cell 2004;118:1-2.

-28 Benhamo R, Efroni S: MicroRNA regulation of molecular pathways as a generic mechanism and as a core disease phenotype. Oncotarget 2015;6:1594-1604.

29 Wang Q, Zhu Y: Epidemiological investigation of constitutional types of Chinese medicine in general population: base on 21948 epidemiological investigation data of nine provinces in China. China J Tradit Chin Med Pharm 2009;24:7-12.

30 Tomasi TE, Horwitz BA: Thyroid function and cold acclimation in the hamster, Mesocricetus auratus. Am J Physiol 1987;252:E260-267.

-31 Carvalho SD, Kimura ET, Biano AC, Silva JE: Central role of brown adipose tissue thyroxine 5'-deiodinase on thyroid hormone-dependent thermogenic response to cold. Endocrinology 1991;128:2149-2159.

-32 Wang Q, Yao S: Molecular basis for cold-intolerant Yang-deficient constitution of Traditional Chinese Medicine. Am J Chin Med 2008;36:827-834.

33 Dauncey MJ: Thyroid hormones and thermogenesis. Proc Nutr Soc 1990;49:203-215. 